Research Article

\title{
Loss Simulation Analysis and Optimization of U-Groove Leaky Coaxial Cable
}

\author{
Junguo Gao, ${ }^{1}$ Zhipeng $M a,{ }^{1}$ Wenhua Yang, ${ }^{2}$ and Xiaohong Zhang $\mathbb{D}^{1}$ \\ ${ }^{1}$ Key Laboratory of Engineering Dielectrics and Its Application, Ministry of Education, \\ Harbin University of Science and Technology, Harbin 150080, China \\ ${ }^{2}$ Xinya Electronic Co. Ltd., Wenzhou 325603, China \\ Correspondence should be addressed to Xiaohong Zhang; x_hzhang2002@hrbust.edu.cn
}

Received 18 November 2019; Accepted 25 May 2020; Published 20 June 2020

Academic Editor: Jit S. Mandeep

Copyright (C) 2020 Junguo Gao et al. This is an open access article distributed under the Creative Commons Attribution License, which permits unrestricted use, distribution, and reproduction in any medium, provided the original work is properly cited.

Leakage coaxial cable is a kind of coaxial cable with various slot structures on the outer conductor of the cable. It can transmit signal and transmit or receive signal and has dual functions of signal transmission line and antenna. Leakage cable has the advantages of strong environmental adaptability, uniform signal coverage, and less attenuation. It is not only widely used in closed or semiclosed space with high signal reception quality, such as tunnels, subway, underground parking lot, and elevators but also can realize security and theft-proof monitoring and protection in some areas, such as oil wells, mining fields and natural resource protection areas, military fortresses, museums, airports, banks, and schools. This paper introduces the classification and electrical parameters of leaky coaxial cables. On the basis of U-groove leaky coaxial cables, the relationship between the parameters of groove holes in U-groove and the loss of leaky coaxial cables is simulated and analyzed by HFSS software. The improved method of U-groove structure is obtained, and the curved hook-groove leaky coaxial cables are designed according to this method. The simulation results show that the coupling loss of the cable is lower than that of the U-groove leakage cable, and the transmission loss is still within the national standard. It lays a theoretical foundation for the design and development of leaky coaxial cable with low coupling loss.

\section{Introduction}

Leakage coaxial cable is abbreviated as leaky cable, which is developed on the basis of radio-frequency coaxial cable [1]. It is a new type of antenna feeder, which not only transmits signals but also has the function of the antenna. Leaky cables have many advantages, such as uniform signal coverage, low price, simple structure, and strong adaptability. In complex environments especially where mobile communication cannot be achieved, the use of leaky cables can ensure communication quality and achieve real-time communication $[2,3]$. Due to its dual functions, leaky coaxial cable has the advantage that a discrete antenna cannot be replaced in the wireless communication system. In recent years, it has been widely used in closed or semiclosed areas such as metros, tunnels, underground shopping malls, mines, underground streets, and large buildings where the electromagnetic wave cannot reach or propagate badly, so as to solve the problem of signal coverage that many single antennas cannot solve $[4,5]$.

If leaky coaxial cables are classified according to electromagnetic radiation, they can be divided into two categories: coupled leaky cables and radiated leaky cables. The slot spacing of the outer conductor of the coupled leakage cable is not in an order of magnitude [6-8] with the wavelength of the electromagnetic wave being transmitted by the cable. Its transmission frequency band is wide, and it is suitable for indoor occasions with a small area. Compared with the coupled leaky coaxial cable, the slot spacing of the outer conductor of the radiation leaky coaxial cable is in the same order of magnitude as the wavelength of the electromagnetic wave signal transmitted by the cable, and the slot spacing is generally equal to the wavelength of the electromagnetic wave or half of the wavelength [9]. Radiation leakage cables are suitable for use in closed or 
semiclosed space such as underground tunnels, mines, and railways where electromagnetic wave signals are difficult to transmit. They are used to enhance electromagnetic wave radiation.

Through experimental analysis of the factors affecting the loss of leaky cables, Jin found that the diameter of the inner conductor of leaky cables is inversely proportional to the attenuation, but when the inner conductor increases to a certain extent, the attenuation is almost unaffected by it [10]. Feng et al. studied the influence of slot parameters of U-groove leaky cable on the usage frequency band, VSWR, and loss of the leaky coaxial cable. It was found that when the pitch of the slot hole decreased, the peak frequency of standing wave and peak frequency of attenuation moved toward high frequency [11]. Through HFSS simulation, Wang et al. analyzed the influence of U-groove pitch and slot size on leakage cable loss and proved that slot pitch size is proportional to leakage cable coupling loss [12]. In this paper, the influence of parameters of U-groove on transmission loss and coupling loss of leaky cable is simulated and analyzed by HFSS software. The structure of U-groove is improved through comparative analysis, and the leaky coaxial cable with a curved hook groove with lower coupling loss is designed.

\section{Electrical Parameters of the Leaky Cable and HFSS Simulation Software}

Leakage cable has three main electrical parameters: characteristic impedance, attenuation constant, and coupling loss [13].

2.1. Characteristic Impedance. Characteristic impedance is an important electrical parameter to characterize the characteristics of leaky coaxial cable. The matching of characteristic impedance has a great influence on the normal operation of leakage cable [14]. The formula for calculating the characteristic impedance of the RF coaxial cable is as follows:

$$
Z_{0}=\sqrt{\frac{R+j \omega L}{G+j \omega C}}=\sqrt{\frac{L}{C}}=\frac{60}{\sqrt{\varepsilon_{r}}} \ln \frac{D}{d}=\frac{138}{\sqrt{\varepsilon_{r}}} \lg \frac{D}{d} .
$$

where $R, L, G$, and $C$ are the primary parameters of RF coaxial cables. For signal transmission cables, the values of $R$ and $G$ are generally much smaller than those of $\omega L$ and $\omega C$, so the values of $R$ and $G$ can be neglected in formula (1). $D$ and $d$ represent the equivalent diameter of the outer conductor and the inner conductor of the RF coaxial cable in millimeters, respectively. At present, the most widely used characteristic impedance of leakage cable is $50 \Omega$ and $75 \Omega$.

2.2. Attenuation Constant. Attenuation constant is a physical quantity that characterizes the loss of an electromagnetic wave signal in the transmission process of leaky cable. It is an important parameter to evaluate the performance of the leaky cable. There are three parts of leakage cable that can cause loss: internal and external conductors, insulating media, and radiation attenuation [15]. In general, the decay constant is expressed by $\alpha_{c}$, and its formula is as follows:

$$
\alpha=\alpha_{c}+\alpha_{d}+\alpha_{r}
$$

In formula (2), $\alpha_{c}$ represents the attenuation constant of the conductor of the leaky cable, which is generated by the conductor inside and outside the leaky cable. $\alpha_{d}$ represents the dielectric attenuation constant of the leaky cable, which is generated by the insulating medium of the leaky cable. $\alpha_{r}$ represents the radiation attenuation constant of the leaky cable, which is generated at the groove of the conductor outside the leaky cable.

In formula (3), $f$ is the frequency of transmitting electromagnetic wave signal, $D$ is the equivalent diameter of the conductor outside the leaky cable, and $d$ is the equivalent diameter of the conductor inside the leaky cable. When the inner and outer conductors of leaky cables are corrugated copper tubes, $K_{1}$ and $K_{2}$ are the resistance increasing coefficients of the inner and outer conductors of leaky cables. When the inner and outer conductors use smooth copper tubes or can be equivalent to smooth copper tubes, $K_{1}$ and $K_{2}$ can be regarded as $1 . \lambda$ denotes the wavelength of an electromagnetic wave propagating in the leaky cable, and $r$ denotes the linear distance between the half-dipole collector antenna and the leaky cable. When the leaky cable is in the single-mode radiation state, the radiation power of the cable is uniform. At this time, the radiation attenuation constant $\alpha_{r}$ of the cable can be calculated according to the coupling loss $L_{c}$ of the leaky cable:

$$
\left\{\begin{array}{l}
\alpha_{c}=\frac{R}{2} \sqrt{\frac{C}{L}=\frac{R}{2 Z_{0}}}=\frac{2.61 \times 10^{-3} \sqrt{f \varepsilon_{r}}}{\lg (D / d)}\left(\frac{K_{1}}{d}+\frac{K_{2}}{D}\right), \\
\alpha_{d}=9.1 \times 10^{-5} f \sqrt{\varepsilon_{r}} \tan \delta_{r}, \\
L_{c}=53.2-10 \lg \frac{\lambda^{2}}{r}-10 \lg \alpha_{r} .
\end{array}\right.
$$

2.3. Coupling Loss. The coupling loss is the electrical parameter that represents the radiation capability of the leaky cable, and it is the only index to distinguish the leaky cable from the RF coaxial cable. The parameters of coupling loss determine the electromagnetic wave energy radiation intensity and radiation range of leakage cable. It is defined as the ratio of the electromagnetic wave signal in the leaky cable to the electromagnetic wave signal coupled by the half-wave coupler antenna at a certain distance from the leaky cable (usually 2 meters).

The calculation formula of coupling loss $L_{c}$ is as follows [16]:

$$
L_{c}=-10 \lg \left(\frac{P_{r}}{P_{t}}\right)
$$

In formula (4), $P_{r}$ denotes the electromagnetic wave power received by the half-dipole collector antenna 2 meters 
from the vertical direction of the leaky cable, and $P_{t}$ denotes the transmission power of the electromagnetic wave in the leaky cable opposite the half-dipole collector antenna.

HFSS (high-frequency structure simulator) is used to simulate and analyze the leaky coaxial cable. HFSS is a three-dimensional electromagnetic simulation software developed by Ansoft company. It is the first commercial three-dimensional electromagnetic simulation software in the world. HFSS has a powerful antenna design function, which can calculate various antenna parameters, such as gain, directivity, and far-field 3D map. The algorithm of HFSS is mainly based on the finite element method. First, the functions corresponding to the boundary value problem and its equivalent variational problem are found out. The part to be solved is decomposed into several small elements, which are connected by nodes. The regional discrete mode will affect the simulation time and accuracy. The unknown quantity inside the element is obtained by selecting the difference value of the node value of the element. The equation between the node quantity is established by the balance relation and the energy relation. Each element equation is combined into a group of algebraic equations and solved by the boundary conditions; thus, the required parameters can be obtained.

In HFSS, when the inner conductor is a corrugated copper pipe, the inner conductor can be equivalent to a solid smooth copper column. The relative permittivity and loss tangent of the insulating medium need to be input manually. The corresponding cylinder is drawn according to the outer diameter of the insulating medium of the leaky cable, and the Boolean subtraction operation is performed with the inner conductor. In the simulation model, the outer conductor is equivalent to a smooth plane without thickness, and a straight line is drawn on the outer surface of the medium. It is drawn by rotating $360^{\circ}$ along the surface of the leaky cable with sweep operation, and its material is set as copper. The plane of slots is drawn according to the slot parameters of the leaky cable, the plane is stretched into a body along the radial direction of the leaky cable and fully intersected with the outer conductor, and then Boolean subtraction operation is done, so the shape of slots can be dug out on the plane of the outer conductor. A half-wave dipole antenna is drawn two meters away from the leaky cable. The conductor is a cylinder with the same length. The length depends on the working frequency wavelength of the simulated leaky cable. The feeding port in the middle is a rectangular plane.

Then, the boundary conditions and excitation methods are set. In the finite element solution, setting the boundary conditions at infinity will seriously affect the solution efficiency. Therefore, an airbox $1 / 4$ wavelength is drawn away from the leaky cable on the surface of the leaky cable; a fanshaped airbox is drawn along the direction of the dipole antenna on the surface of the box; it is the radiation boundary of the leaky cable.

\section{Loss Simulation Analysis of the U-Groove Leakage Cable}

HFSS simulation software is used to analyze the influence of leakage cable parameters on transmission loss and coupling loss.
3.1. Basic Parameters of the U-Groove Leakage Cable. Taking 50-42 U-groove leaky coaxial cable as an example, the characteristic impedance of the leaky cable is $50 \Omega$. The inner conductor is made of a corrugated copper tube. The outer diameters of the corrugated copper tube trough and wave peak are $14.5 \mathrm{~mm}$ and $17.3 \mathrm{~mm}$, respectively, and the rolling pitch is $10.1 \mathrm{~mm}$. Physical foaming insulation is chosen as the dielectric material. The physical foaming degree is $78 \%$, and its outer diameter is $42.5 \mathrm{~mm}$. The relative dielectric constant of the insulating medium is 1.245 , and the tangent value of the loss angle is $1.7 \times 10^{-5}$. The outer conductor is made of a rolled copper strip with an outer diameter of $43.8 \mathrm{~mm}$ and a width of $150 \mathrm{~mm}$. The groove pattern is shown in Figure 1. The groove width $a$ is $13 \mathrm{~mm}$ perpendicular to the axial direction, the overall groove width $b$ is $11 \mathrm{~mm}$, the axial groove width $c$ is $3.5 \mathrm{~mm}$, the overall groove length $d$ is $77 \mathrm{~mm}$, the adjacent two U-grooves are opposite, the groove edge is rounded, and its radius is $2 \mathrm{~mm}$.

According to the theory of space harmonic radiation, the leakage cable will generate infinite space harmonics because of periodic slots on the outer conductor. When the harmonic order $n$ is less than or equal to 1 , the space harmonics generated are radiated waves. When $f$ is greater than $f_{1}$ and less than $2 f_{1}$, the spatial harmonics of the 1st mode are radiated waves, and the leaky cable is in the single-mode radiation state. At this time, the electromagnetic radiation effect of the leaky cable is the best. Therefore, the working frequency of leakage cable is taken as the central frequency, and the cycle $p$ of U-groove is calculated between $118.5 \mathrm{~mm}$ and $237.1 \mathrm{~mm}$, so the cycle $p$ of U-groove is $200 \mathrm{~mm}$.

\subsection{Establishment of Simulation Model for the U-Groove} Leakage Cable. Because the inner conductor is corrugated copper tube structure and the outer conductor is rolled copper strip longitudinal cladding structure, the simulation model should be simplified. In order to obtain more accurate simulation data, according to previous simulation experience, the inner conductor can be equivalent to a smooth copper column. In each cycle of the inner conductor, peak and trough are processed according to a certain proportion of duty cycle, which is generally $3: 17$. When calculating the equivalent inner diameter of the copper tube, the average inner diameter of wave crest and trough cannot be directly used but calculated by weighting. Equivalent outer diameter is usually calculated by

$$
d=0.85 d_{1}+0.15 d_{2}-t .
$$

Among them, $d$ is the inner conductor equivalent smooth copper tube diameter, $d_{1}$ is the inner conductor peak outer diameter, $d_{2}$ is the inner conductor trough outer diameter, and $t$ is the inner conductor tube wall thickness. According to the wave crest and trough of the inner conductor, the outer diameter of the copper column is $16.88 \mathrm{~mm}$.

In the actual measurement process of leakage cable coupling loss, leakage cable with a length of $50 \mathrm{~m}$ is usually selected as the experimental sample for measurement. However, in the process of simulation and analysis, due to 
the limited computing power of the computer used in the simulation, when the leakage cable model is too long, the computer simulation time is too long, and the computer system collapses. Therefore, it is necessary to choose the appropriate length not only to reduce the operating pressure of the simulation computer but also to accurately get the loss value of leakage cable. Therefore, the cycle of U-grooves in the leaky cable is taken as the reference length. The length of the model increases with the increase in the number of $\mathrm{U}$-grooves. The coupling loss value at the center of the leaky cable model is obtained by simulation. Figure 2 shows the variation curve between the central coupling loss and the number of cycles in the leaky cable model. It can be seen that when the number of cycles equals 10, the value of coupling loss has become stable, so the length of the U-groove leakage cable model can be determined to be $2023 \mathrm{~mm}$.

The U-groove is drawn according to the parameters shown in Figure 1. The working frequency is set to $900 \mathrm{MHz}$, and the groove model is shown in Figure 3.

\subsection{Analysis of Simulation Data of the U-Groove Leakage} Cable. Because the coupling loss of the leaky cable varies with the axial coordinates of the leaky cable, it is necessary to use multiple test points to simulate and calculate the coupling loss of the leaky cable. In the actual test process of leakage cable coupling loss, when the length of the test cable is 60 meters, 5 meters away from both ends of the leakage cable does not participate in the measurement process. Therefore, according to the length of the model cable, the simulation process is not involved at $168.6 \mathrm{~mm}$ between the two ends of the simulation model. Therefore, a half-wave couple collector antenna is designed to move from $500 \mathrm{~mm}$ to $1500 \mathrm{~mm}$ in the axial direction of the leaky cable.

The vertical distance between the half-dipole collector antenna and the leaky cable is set at $2 \mathrm{~m}$, and the coupling loss data are obtained by simulation as shown in Figure 4. $L_{c, 50}$ and $L_{c, 95}$ of the hook-groove leakage cable are $66.77 \mathrm{~dB}$ and $72.86 \mathrm{~dB}$, respectively. The calculation shows that the transmission loss of leaky coaxial cable is $2.32 \mathrm{~dB} / 100 \mathrm{~m}$. The measured data of the same type of $U$-groove loss in a factory are transmission loss of $2.36 \mathrm{~dB} / 100 \mathrm{~m}$ and coupling loss of $71.4 \mathrm{~dB}$. The error between the simulation results and the measured results is very small, and both meet the national standards. The model can be used to continue the simulation.

The relationship between the vertical and axial slot width $a$ of $\mathrm{U}$-groove and the loss of leakage cable is shown in Figure 5. The transmission loss of leakage cable increases linearly with the increase of $a$ in the range of $10 \mathrm{~mm}-16 \mathrm{~mm}$, and the coupling loss is inversely proportional to the slot width $a$. When $a$ is changed between $11 \mathrm{~mm}$ and $15 \mathrm{~mm}$, the coupling loss of the leaky cable changes very little, and the difference between the maximum and the minimum is only about $0.3 \mathrm{~dB}$. The simulation results show that the transmission loss of U-groove leaky cable increases by $4.3 \times 10^{-3} \mathrm{~dB} / 100 \mathrm{~m}$ and the coupling loss decreases by $0.225 \mathrm{~dB}$ with an increase of $1 \mathrm{~mm}$ of vertical axial groove width.
The relationship between groove width $b$ and leakage cable loss is shown in Figure 6. When $b$ exceeds $12 \mathrm{~mm}$, the transmission loss of $\mathrm{U}$-groove exceeds $2.4 \mathrm{~dB} / 100 \mathrm{~m}$, and the coupling loss does not exceed the standard in the range of $8 \mathrm{~mm}-14 \mathrm{~mm}$. The transmission loss is proportional to slot width $b$, and the coupling loss is inversely proportional to slot width $b$. The slope of the transmission loss curve increases with the increase of the overall slot width, and the coupling loss curve fluctuates when $b=11 \mathrm{~mm}$. According to the calculation results, when the overall width of $U$-groove increases by $1 \mathrm{~mm}$, the transmission loss of $\mathrm{U}$-groove leaky cable increases by $0.11 \mathrm{~dB} / 100 \mathrm{~m}$, and the coupling loss decreases by $1.75 \mathrm{~dB}$.

The relationship between the axial groove width $c$ of $\mathrm{U}$-groove and the loss of leakage cable is shown in Figure 7. When $c$ exceeds $4.5 \mathrm{~mm}$, the coupling loss increases from $72.59 \mathrm{~dB}$ to $79.29 \mathrm{~dB}$, which exceeds the standard value of $74 \mathrm{~dB}$. The transmission loss of the leaky cable decreases with the increase of the axial groove width $c$, and the coupling loss is opposite to that of the leaky cable. The calculation shows that the transmission loss of $\mathrm{U}$-groove leakage cable decreases by $0.033 \mathrm{~dB} / 100 \mathrm{~m}$ and the coupling loss increases by $3.94 \mathrm{~dB}$ for each increase of the axial groove width of U-groove by $1 \mathrm{~mm}$. Compared with Figure 5, it is found that the influence of axial slot width $c$ on cable loss is greater than that of vertical slot width $a$, especially coupling loss.

The relationship between the overall groove length $d$ of $\mathrm{U}$-groove and the loss of leakage cable is shown in Figure 8. The transmission loss increases with the increase of the groove length, while the coupling loss decreases with the increase of the groove length, but the fluctuation is large. According to the simulation data, the transmission loss of U-groove leakage cable increases by $6.84 \times 10^{-3} \mathrm{~dB} / 100 \mathrm{~m}$ and the coupling loss decreases by $0.407 \mathrm{~dB}$ with the increase of the overall groove length of U-groove by $1 \mathrm{~mm}$.

The relationship between the cycle $p$ of $U$-groove and the loss of leakage cable is shown in Figure 9. The transmission loss of leakage cable is inversely proportional to the period of $\mathrm{U}$-groove, and the coupling loss is proportional to the period. When the cycle exceeds $212 \mathrm{~mm}$, the coupling loss has exceeded the standard value. Periodic variation has little effect on transmission loss of leaky cables. The difference between the maximum and minimum transmission loss in known simulation data is only $0.03 \mathrm{~dB} / 100 \mathrm{~m}$. The transmission loss of $\mathrm{U}$-groove leakage cable decreases by $4.42 \times 10^{-4} \mathrm{~dB} / 100 \mathrm{~m}$ and the coupling loss increases by $0.21 \mathrm{~dB}$ with the increase of the cycle of $\mathrm{U}$-groove by $1 \mathrm{~mm}$. Therefore, when the transmission loss of leaky cables is low, it can be approximated that the slot cycle of leaky cables only affects the coupling loss of cables.

For U-grooves, the size of the axial groove width is the main determinant of their loss characteristics, and its main function is to destroy the symmetry of the electric field components. Compared with the vertical axial groove width, the axial groove width has a greater impact on the loss of U-groove leakage cable. In known data, the overall groove width has the greatest impact on the transmission loss of $\mathrm{U}$-groove leakage cable, and the axial groove width has the greatest impact on the coupling loss of leakage cable. In 


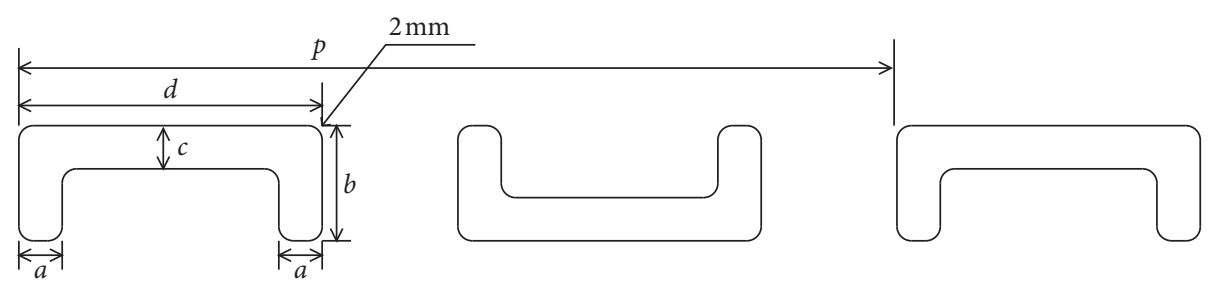

Figure 1: Diagram of U-groove.

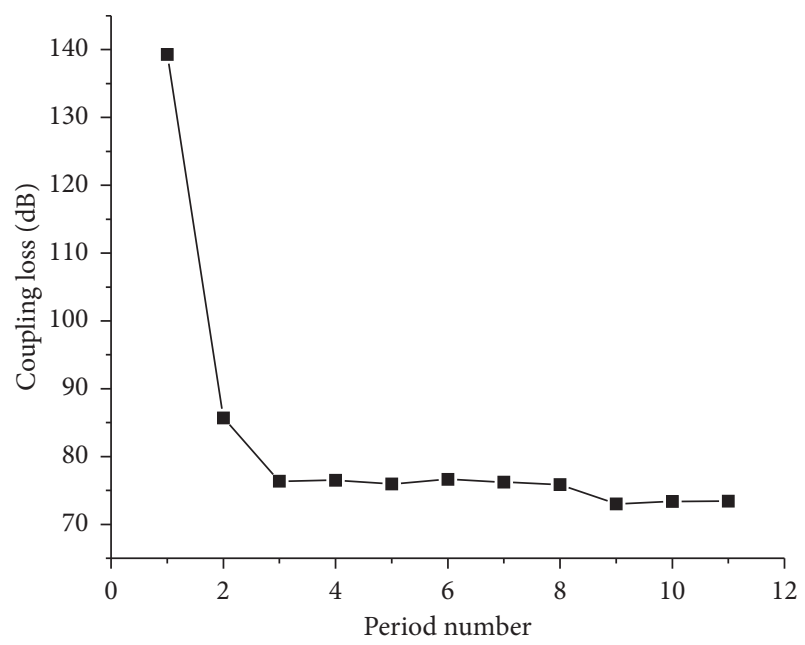

Figure 2: Relation between central coupling loss and period number of U-grooves in the model.

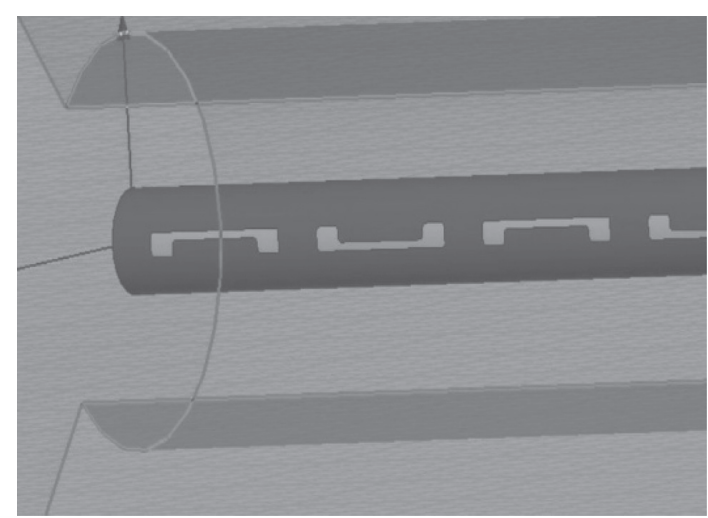

FIGURE 3: Schematic diagram of a U-groove leakage cable simulated groove.

order to reduce the coupling loss of U-groove leakage cable, the axial groove width of $U$-groove can be reduced, and the vertical axial groove width of the groove hole can be increased. In order to reduce the transmission loss of leakage cable, the overall groove width of $U$-groove should be reduced first.

\section{Loss Simulation Analysis of the Curved Hook- Groove Leakage Cable}

According to the influence factors of loss of U-groove leaky coaxial cable, the curved hook-groove leaky coaxial cable is

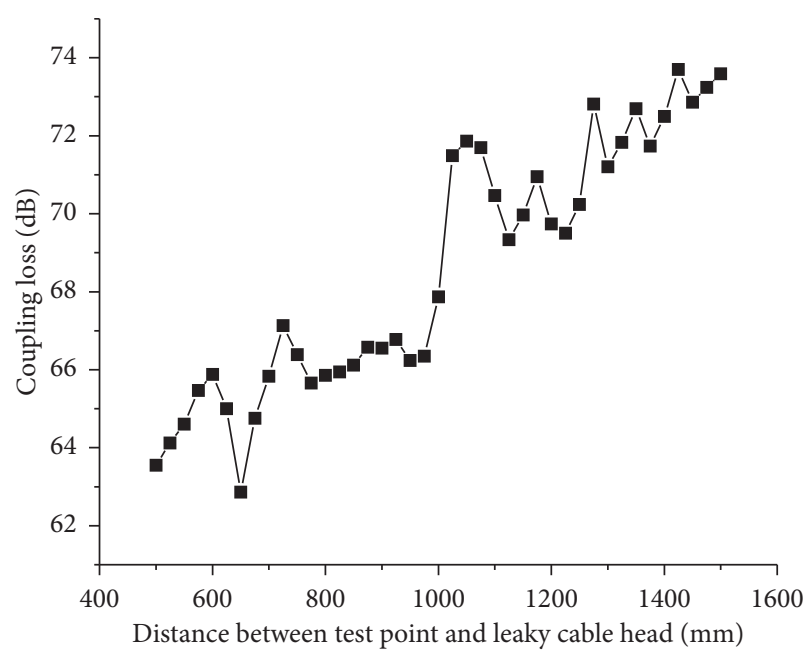

FIGURE 4: Coupling loss of U-groove leakage cable.

designed. Compared with U-groove, the axial component of the groove is increased. In order to reduce the transmission loss of cable, the groove in the vertical and axial direction of half side is removed.

4.1. Basic Parameters of the Curved Hook-Groove Leakage Cable. The characteristic impedance of the leaky coaxial cable is $50 \Omega$. The outer diameter of the inner and outer conductors and the insulating material are the same as those of the U-grooves mentioned above. The groove pattern is shown in Figure 10. The overall length of the groove hole $a$ is $80 \mathrm{~mm}$, and the upper edge length $b$ is $30 \mathrm{~mm}$. Axial groove width $c$ is $3 \mathrm{~mm}$, vertical groove width $d$ is $13 \mathrm{~mm}$, and overall groove width $e$ is $11.5 \mathrm{~mm}$. The two adjacent hook grooves are symmetrical with a cycle $p$ of $190 \mathrm{~mm}$ and a circular corner with a radius of $2 \mathrm{~mm}$ at the edge of the grooves.

The inner conductor is equivalent to a smooth copper column with an inner diameter of $16.88 \mathrm{~mm}$, and the overall length of the leakage cable model is $2230 \mathrm{~mm}$. The method of establishing the model is the same as that of U-groove. The groove model is shown in Figure 11.

4.2. Analysis of Simulation Data of the Curved Hook-Groove Leakage Cable. The simulation results show that the coupling loss data are shown in Figure 12. $L_{c, 50}$ and $L_{c, 95}$ of the curved hook-groove leakage cable are $64.85 \mathrm{~dB}$ and $68.14 \mathrm{~dB}$, 


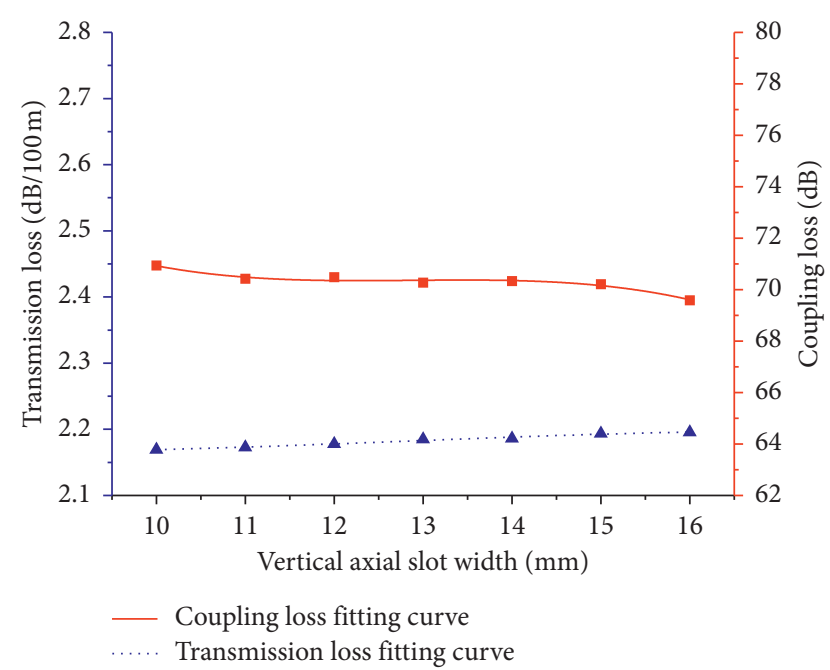

Figure 5: The relation between the width of U-groove in the vertical axial direction and the loss of leakage cable.

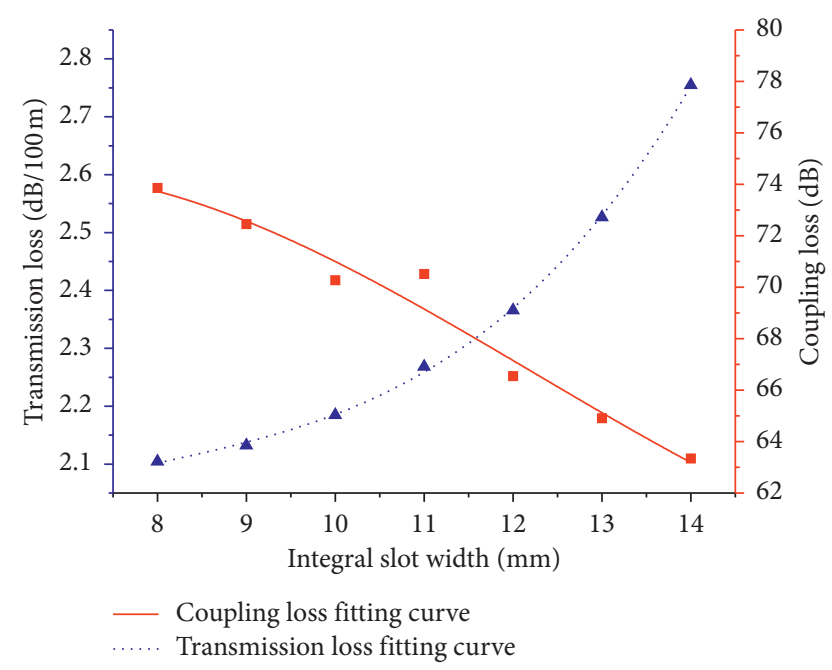

FIGURE 6: The relationship between the overall groove width of $U$ groove and cable leakage loss.

respectively, which are much lower than the national standard value of $74 \mathrm{~dB}$.

The influence of the overall groove length of U-groove and curved hook-groove on transmission loss and coupling loss of leakage cable is shown in Figures 13(a) and 13(b). With the increase of the axial component of the curved hook-groove, the coupling loss of the leaky cable decreases greatly, but the transmission loss of the cable also increases. The transmission loss of the cable with curved hook-groove leakage is proportional to the groove length, while the coupling loss is the opposite. In the range of simulation parameters, the transmission loss of the curved hook-groove leaky coaxial cable increases by about $0.15 \mathrm{~dB} / 100 \mathrm{~m}$, but it is still lower than the national standard value of $2.4 \mathrm{~dB} / 100 \mathrm{~m}$. The coupling loss of the curved hook-groove cable is about $6 \mathrm{~dB}$ smaller than that of the U-groove cable. Compared with the U-groove, the coupling loss of the curved hook-groove leakage cable fluctuates less in the whole groove length of

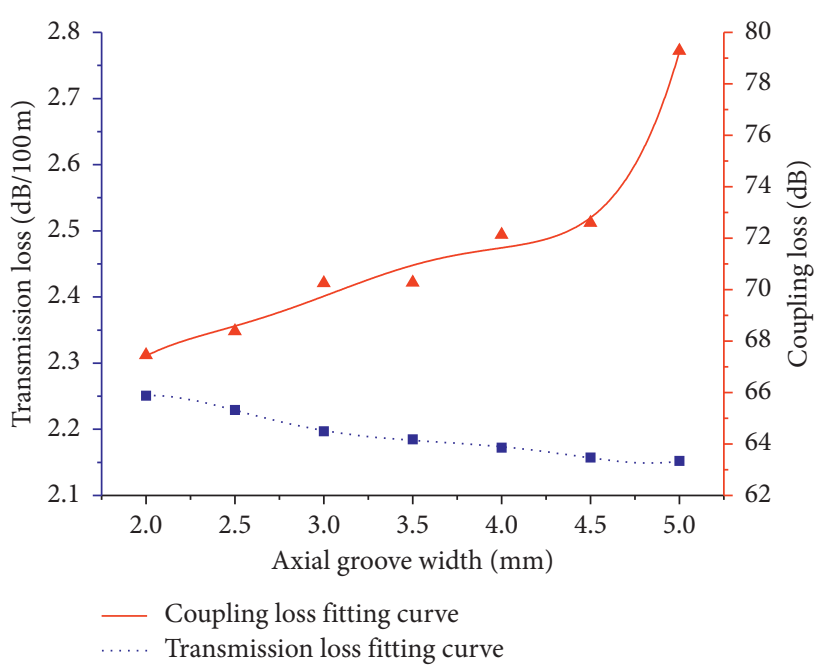

FIgURE 7: The relationship between the axial slot width of U-groove and the loss of leakage cable.

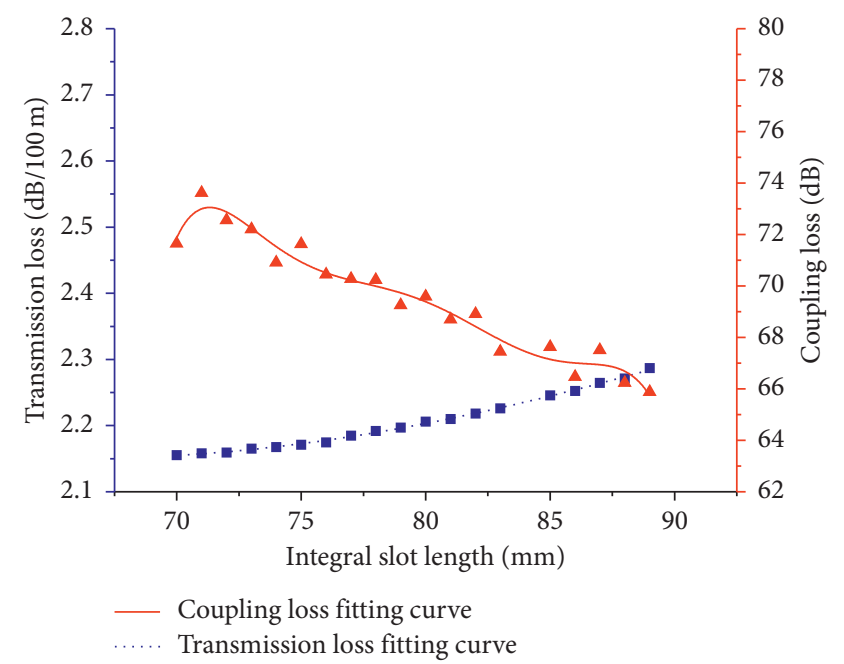

Figure 8: The relationship between the overall groove length of $U$ groove and cable leakage loss.

$70 \mathrm{~mm}-90 \mathrm{~mm}$. The transmission loss of leakage cable increases by $4.7 \times 10^{-3} \mathrm{~dB} / 100 \mathrm{~m}$ and the coupling loss decreases by $0.204 \mathrm{~dB}$ with the increase of slot length of curved hook groove by $1 \mathrm{~mm}$.

The influence trend of upper edge length on the loss of leakage cable is shown in Figure 14. The coupling loss decreases with the increase of the upper edge length. The transmission loss is contrary to its trend, and the slope of the transmission loss curve is almost unchanged. When the length of the upper side of the curved hook-groove exceeds $32 \mathrm{~mm}$, the transmission loss of the leakage cable exceeds the standard value. The transmission loss increases $0.0204 \mathrm{~dB} /$ $100 \mathrm{~m}$ and the coupling loss decreases $0.2 \mathrm{~dB}$ for every $1 \mathrm{~mm}$ increase of the upper edge length of the curved hook groove.

The influence of the axial groove width of $U$-groove and curved hook-groove on transmission loss and coupling loss of leakage cable is shown in Figures 15(a) and 15(b). The influence of the axial groove width on the loss of the leaky 


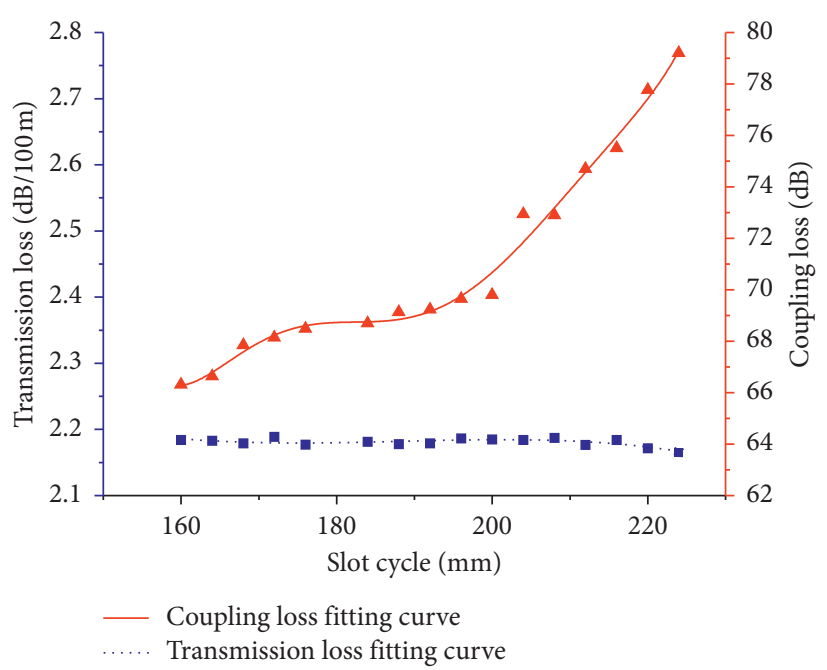

Figure 9: Relation between the cycle of U-groove and the loss of leakage cable.

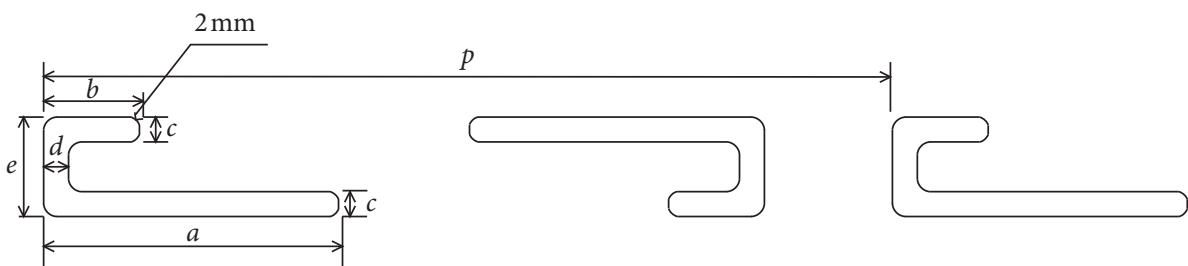

FIgURE 10: Diagram of the size of curved hook-groove.

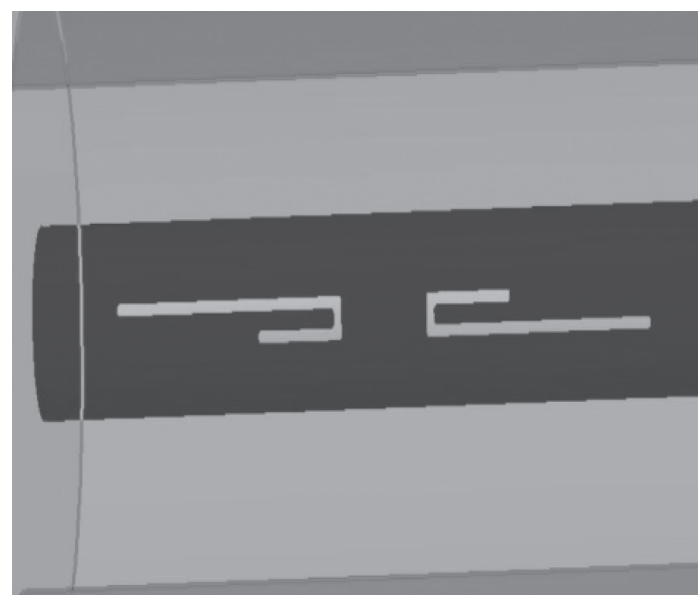

FIGURE 11: Schematic diagram of simulated groove for curved hook-groove leakage cable.

cable is the same. The axial groove width is proportional to the coupling loss and inversely proportional to the transmission loss. When the axial groove width is less than $3 \mathrm{~mm}$, the transmission loss is higher than the national standard value. The coupling loss of curved hook-groove leakage cable is about $6 \mathrm{~dB}$ lower than that of U-groove leakage cable under the same axial groove width. When the axial slot width of the curved hook groove increases by $1 \mathrm{~mm}$, the transmission loss of leaky cable decreases by $0.067 \mathrm{~dB} / 100 \mathrm{~m}$ and the coupling loss increases by $1.33 \mathrm{~dB}$.

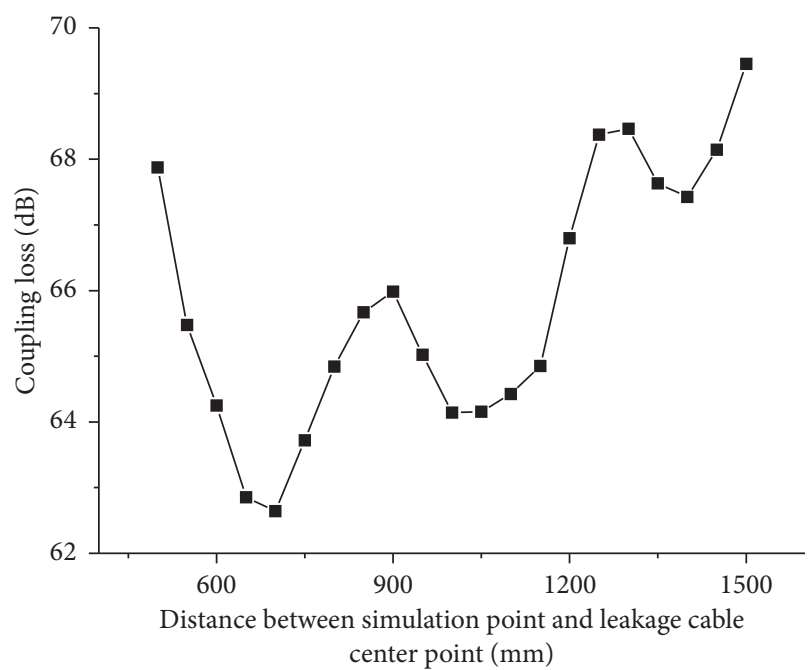

FIGURE 12: Coupling loss of curved hook-groove leakage cable.

The trend of influence of groove width of U-groove and curved hook groove on transmission loss and coupling loss of leakage cable is shown in Figures 16(a) and 16(b). With the increase of groove width, coupling loss decreases linearly and transmission loss increases linearly. When the overall groove width of the curved hook groove exceeds $11 \mathrm{~mm}$, the transmission loss increases rapidly, which directly exceeds the national standard value. Under the same groove width, the coupling loss of curved hook-groove leakage cable is 


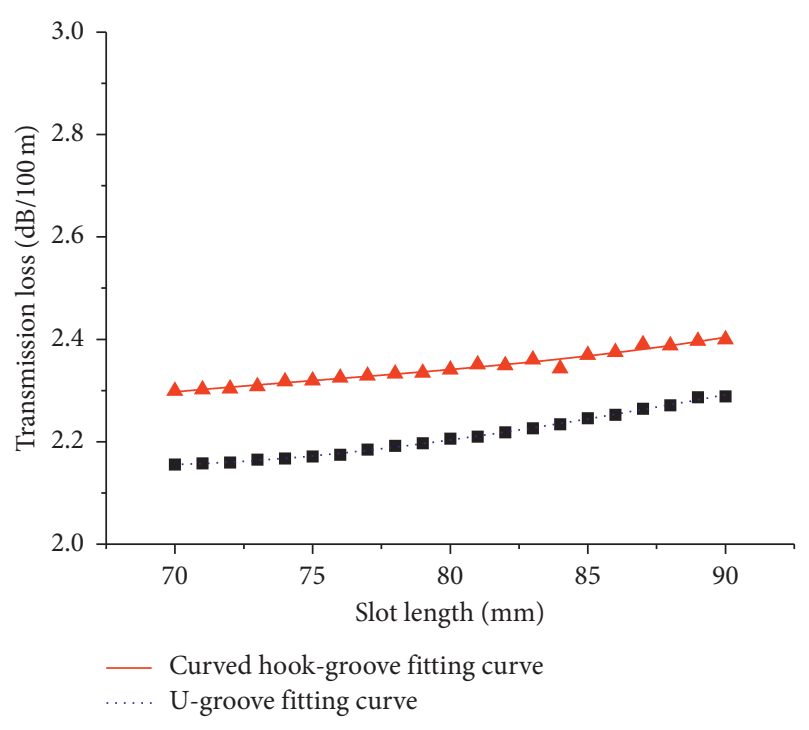

(a)

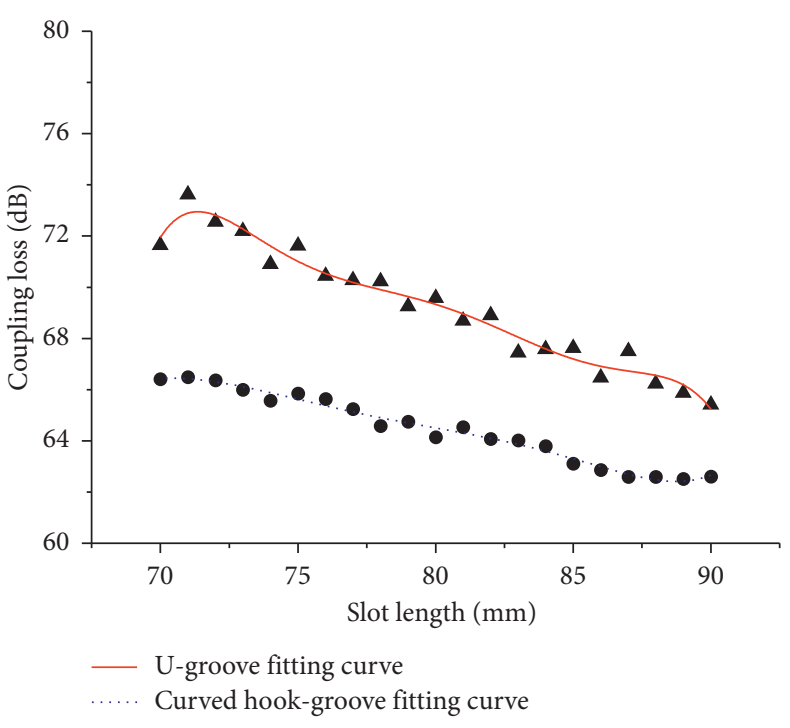

(b)

Figure 13: Trend of influence of overall groove length of U-groove and curved hook groove on leakage cable loss.

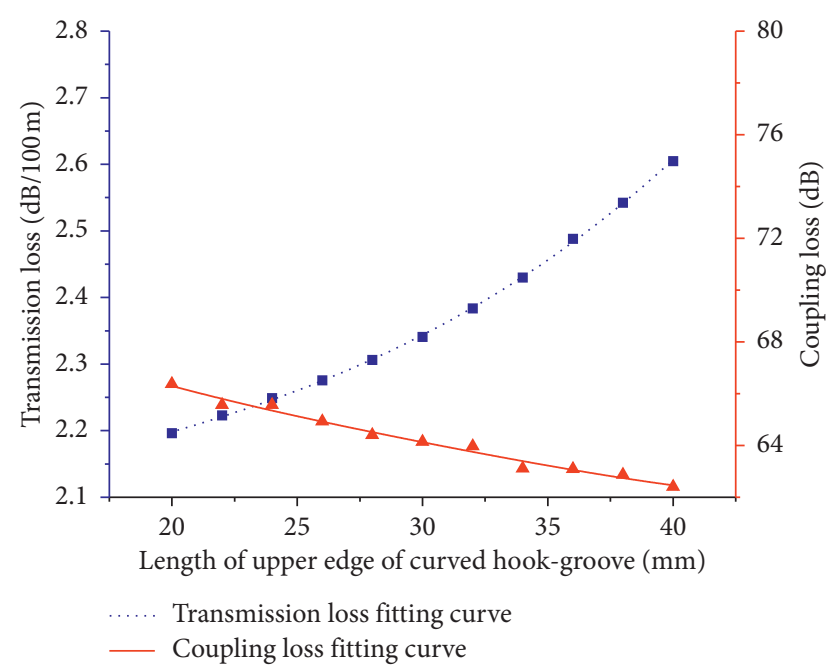

FIGURE 14: Effect of the length of the upper side of the curved hookgroove on the loss of leakage cable.

about $6 \mathrm{~dB}$ lower than that of U-groove leakage cable. The transmission loss of the leaky cable increases by $0.24 \mathrm{~dB} /$ $100 \mathrm{~m}$, and the coupling loss decreases by $1.53 \mathrm{~dB}$ for every $1 \mathrm{~mm}$ increase of the overall groove width of the curved hook groove. Compared with the overall groove length, the overall groove width has a greater impact on leakage loss.

The influence trend of the groove cycle of $\mathrm{U}$-groove and curved hook groove on transmission loss and coupling loss of leaky cable is shown in Figures 17(a) and 17(b). The coupling loss of leaky cable is proportional to the cycle, and the transmission loss is inversely proportional to it. And in the selected groove cycle range, the loss data are in line with the national standards. Compared with the change of groove size, the change of the groove cycle of curved hook-groove leakage cable has less influence on the loss of leakage cable. When the groove cycle varies from $160 \mathrm{~mm}$ to $200 \mathrm{~mm}$, the coupling loss of the curved hook-groove leakage cable is about $4 \mathrm{~dB}$ smaller than that of the U-groove leakage cable. The transmission loss of leaky cable decreases by $2.97 \times 10^{-4} \mathrm{~dB} / 100 \mathrm{~m}$ and the coupling loss increases by $0.045 \mathrm{~dB}$ every $1 \mathrm{~mm}$ of the cycle.

In [12], the author has simulated that the longer the vertical axial groove width of $\mathrm{U}$-groove is, the bigger the transmission loss of leaky cable is, and the smaller the coupling loss is. The longer the axial slot width is, the smaller the transmission loss of the leaky cable is, and the larger the coupling loss is, which is the same as the conclusion of this paper. The data of transmission loss and coupling loss of U-groove leaky cable obtained by simulation in [12] are similar to the data in this paper, while the coupling loss of curved hook-groove leaky cable designed in this paper is significantly lower than that of U-groove, so it can be proved that the design of curved hook groove can effectively reduce the coupling loss of the leaky cable.

In the known data, the slot cycle has the least effect on the loss performance of the curved hook-groove cable, and the overall slot width has the greatest effect on the loss of the leakage cable. Therefore, when it is necessary to adjust the loss performance of curved hook-groove leakage cable, the adjustment of the overall groove width should be considered first.

\section{Analysis of the Relationship between Slot Parameters and Losses}

When the current flows on the leaky cable conductor, the corresponding magnetic field will be generated, and its direction is perpendicular to the current direction. If there is a slot on the outer conductor, the propagation of the current on the outer conductor is restricted, the magnetic field around the cable will also change, and the electromagnetic energy will propagate outward along the slot of the outer conductor. 


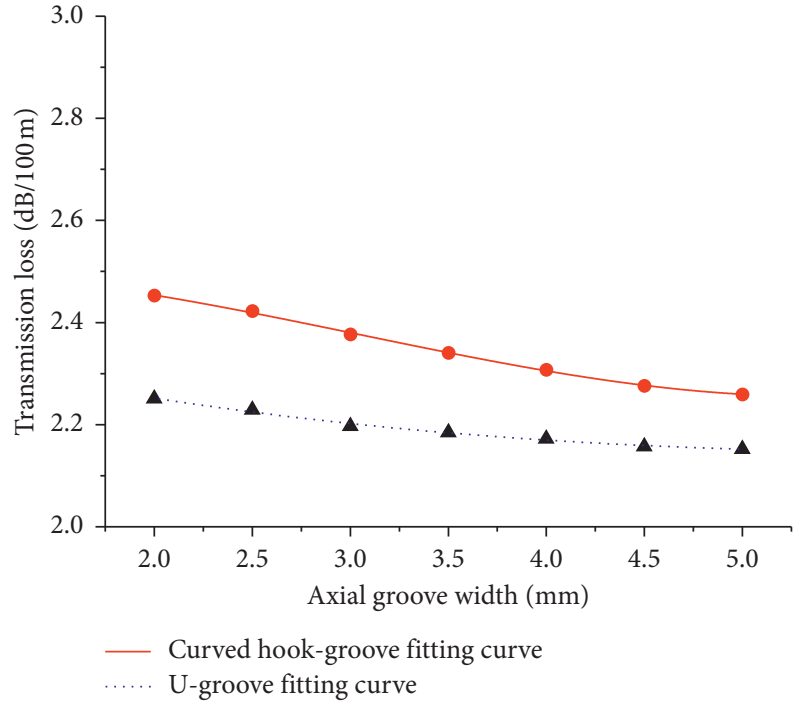

(a)

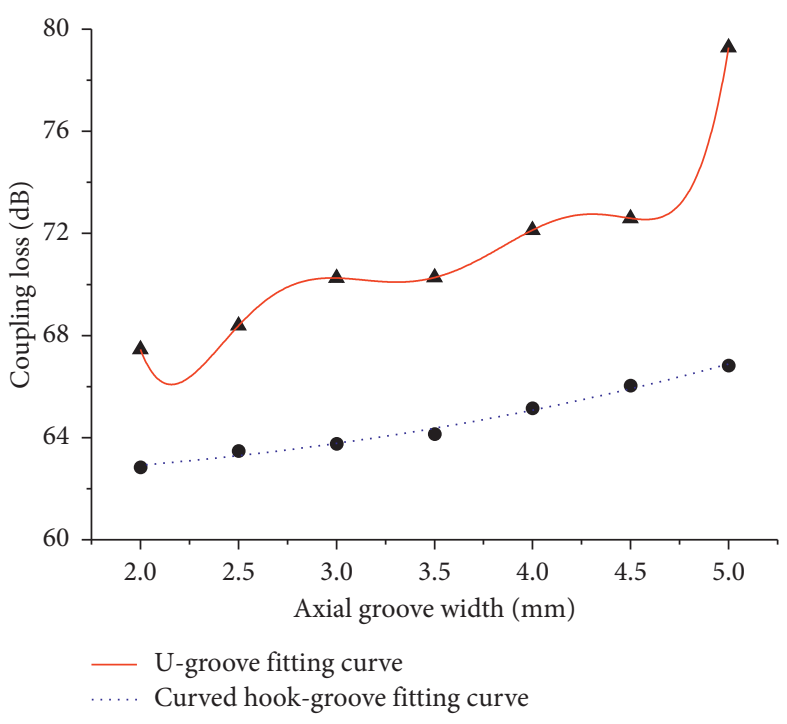

(b)

FIgURE 15: The influence of the axial groove width of U-groove and curved hook groove on the loss of leakage cable.

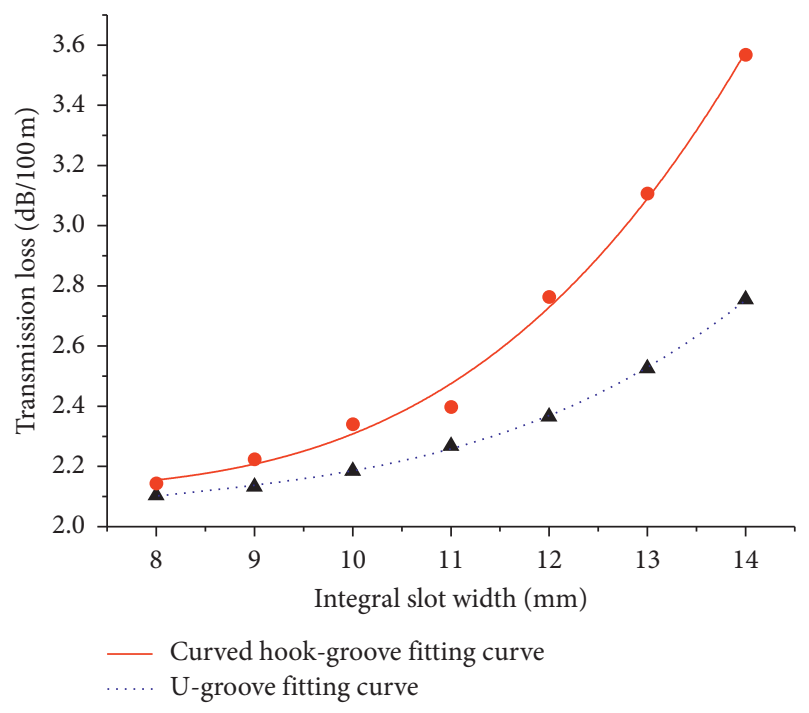

(a)

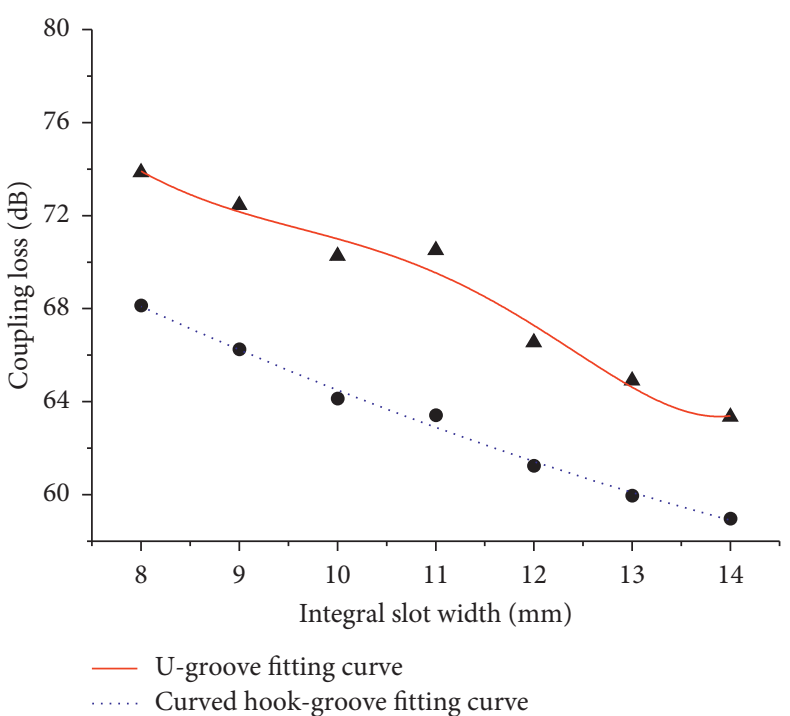

(b)

FIgURE 16: Trend of influence of groove width of U-groove and curved hook groove on the loss of leakage cable.

Transmission loss is composed of conductor attenuation, medium attenuation, and radiation attenuation. Conductor attenuation is mainly produced by the inner conductor, and the outer conductor has little influence on conductor attenuation. It can be approximately considered that changing the size of the outer conductor slot of leaky cable only affects the radiation attenuation of leaky cable. When the overall length and width of U-groove and curved hook-groove increase, the blocking effect of groove on the transmission of external conductor current increases, the electromagnetic wave is more easily transmitted to the outside through the outer conductor groove, and the radiation attenuation of cable increases accordingly, so the transmission loss of leakage cable is directly proportional to the overall length and width of U-groove and curved hook groove. When the radiation attenuation of the leaky cable increases, the electromagnetic wave energy radiated along the slot of the outer conductor of the leaky cable is larger, the electromagnetic wave radiation range will also increase, the electromagnetic wave signal received by the half-wave dipole antenna will be stronger, and the coupling loss of the leaky cable will also be smaller, so the coupling loss of the leaky cable with U-groove and curved hook groove has the opposite trend with the transmission loss.

When the overall slot width of U-groove and curved hook-groove leaky cable is unchanged, and the axial slot width becomes smaller, the vertical axial part of the slot is larger, which has a stronger blocking effect on the current 


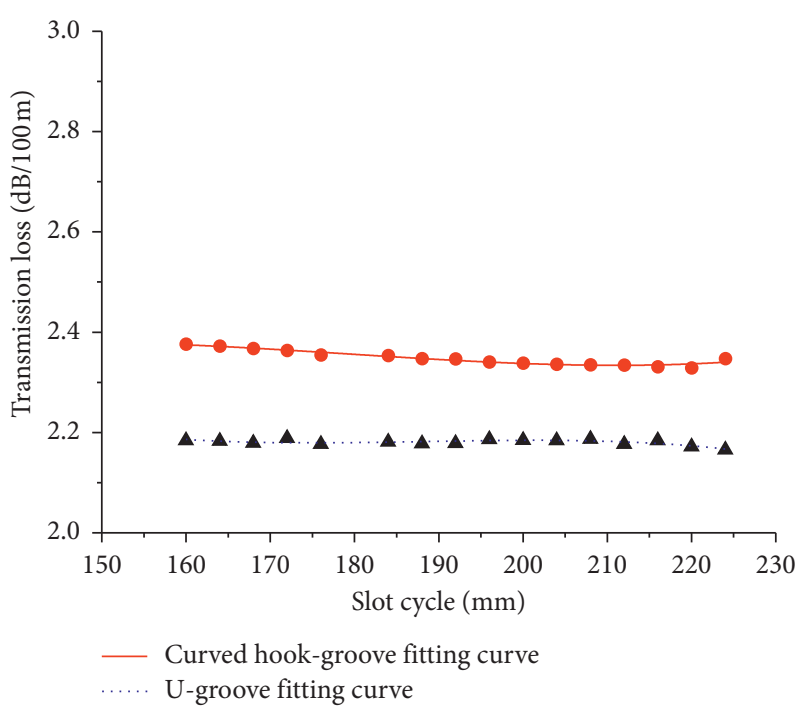

(a)

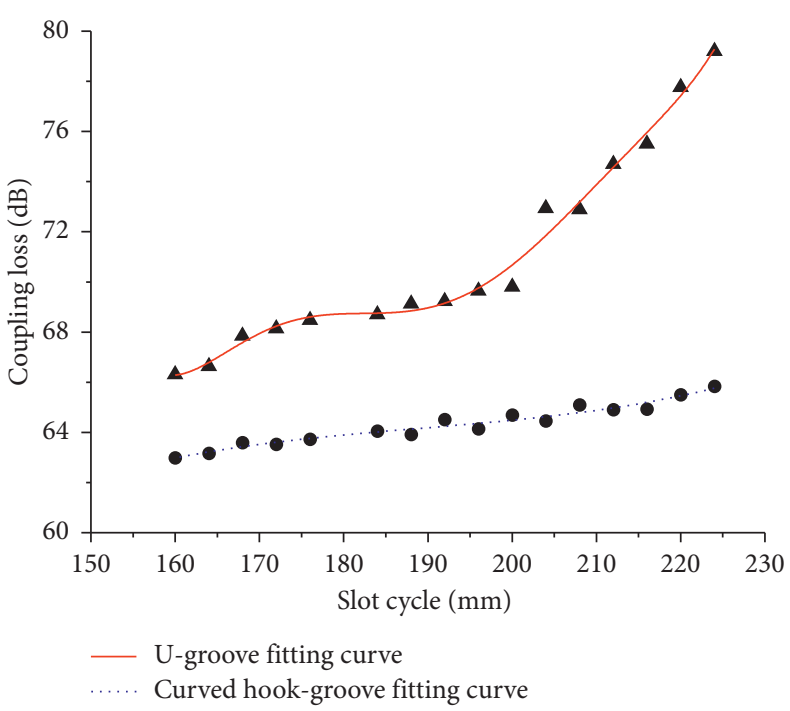

(b)

Figure 17: Trend of influence of groove width of U-groove and curved hook groove on the loss of leakage cable.

transmission along the axial direction, and the electromagnetic wave is easier to spread to the outside, so the coupling loss decreases, and the transmission loss increases with the increase of radiation attenuation.

Each U-groove and each curved hook groove is equivalent to an electromagnetic wave radiation source, and the electromagnetic waves radiated between adjacent slots occur in the phase superposition. The simulation results show that when the slot period is reduced, the number of slots per unit length of cable increases, which is conducive to the phase superposition between electromagnetic waves, and the stronger the directionality of electromagnetic wave propagating to the outside, the stronger the electromagnetic wave signal received by the half-wave dipole antenna, and the less the coupling loss.

\section{Conclusion}

In this paper, the relationship between the slot parameters of $\mathrm{U}$-groove and the loss of leaky coaxial cable is analyzed by HFSS software simulation, which lays a theoretical foundation for the design and development of leaky coaxial cable with low coupling loss. Through the simulation analysis, it is found that the overall slot width has the greatest impact on the transmission loss of U-groove leaky cable, and the axial slot width has the greatest impact on the coupling loss of the leaky cable. The transmission loss increases by $0.11 \mathrm{~dB} / 100 \mathrm{~m}$ for every $1 \mathrm{~mm}$ increase in the overall groove width of $\mathrm{U}$-groove, and the coupling loss increases by $3.94 \mathrm{~dB}$ for every $1 \mathrm{~mm}$ increase in the axial groove width. According to the simulation, the improved method of U-groove structure is obtained. In order to reduce the coupling loss of $U$-groove leaky coaxial cable, the axial slot width of U-groove can be reduced and the vertical axial slot width of the slot can be increased. In order to reduce the transmission loss, we should first consider reducing the overall slot width of the U-groove. According to this method, a kind of leaky coaxial cable with curved hook-groove is designed. Compared with $\mathrm{U}$-groove, the curved hook-groove leaky cable can reduce coupling loss of leaky cable by about $4 \mathrm{~dB}$. The overall slot width has the greatest influence on the loss of the curved hook-groove leaky cable. The transmission loss of the leaky cable increases by $0.24 \mathrm{~dB} / 100 \mathrm{~m}$ and the coupling loss decreases by $1.53 \mathrm{~dB}$ every $1 \mathrm{~mm}$ of the overall slot width.

\section{Data Availability}

No data were used to support this study.

\section{Conflicts of Interest}

The authors declare that there are no conflicts of interest regarding the publication of this paper.

\section{Acknowledgments}

This work was supported in part by the National Natural Science Foundation of China (Grant No. 51577045), Youth Innovation Training Plan of Heilongjiang Universities (UNPYSCT-2015047), and Heilongjiang Postdoctoral Research Start-Up Gold Project (LBH-Q19106).

\section{References}

[1] T. Zhang and J. Chen, "Theory analysis of the leaky communication in underground and discussion on actualizing projects of the system," Metal Mine, vol. 8, pp. 47-50, 2006.

[2] X. Zhang, Z. X. Zhao, X. D. Yang, L. Guo, and W. Li, "Research on the radiation characteristics of leaky coaxial cable and spiral antenna in the blind zone," in Proceedings of the 2006 International Conference on Communications, pp. 811-815, IEEE, Guilin, China, June 2006.

[3] Q. Li, C. Yu, and X. Liu, "Design of radiation leaky coaxial cables," Optical Fiber \& Electric Cable, vol. 38, no. 4, pp. 30-32, 2004. 
[4] M. Huang and H. Lin, "Recent development of leaky coaxial cables," Optical Fiber \& Electirc Cable and Their Applications, vol. 6, pp. 14-17, 1994.

[5] J. H. Wang and K. K. Mei, "Theory and analysis of leaky coaxial cables with periodic slots," IEEE Transactions on Antennas and Propagation, vol. 49, no. 12, pp. 1723-1732, 2001.

[6] X. D. Yang, S. Feng, X. Zhang, X. Ren, and W. Li, "Research of single mode radiation leaky coaxial cable used for ultra wideband communication," in Proceedings of the 2006 International Conference on Communications, Circuits and Systems, pp. 1261-1264, IEEE, Guilin, China, June 2006.

[7] S. Yao and Z. Zhu, "Performance analysis and application of leakage coaxial cable," Coal Mine Machinery, vol. 7, pp. 29-31, 2003.

[8] J. Zhang, Y. Jiang, and Y. Gu, "Research of type SLYFY(N)-5042 leaky coaxial cable," Optical Fiber \& Electric Cable, vol. 1, pp. 33-36, 2005.

[9] Y. Xiao and W. Zhang, "Performance analysis of leaky cables," Mobile Communications, vol. 26, no. 6, pp. 40-42, 2002.

[10] J. Jin, "Design and performance study of leakage coaxial cable for 4G," Design \& Application, vol. 42, no. 4, pp. 55-57, 2016.

[11] J. Feng, X. Liu, and X. Wu, "The influence of grooving parameters on the electrical performance of U-groove radiation leakage cable," Cables \& Optical Cables, vol. 1, pp. 69-71, 2015.

[12] L. Wang, L. Yang, F. Yang et al., "Design and implementation of a U-shaped leaky coaxial cable," Electronic Science \& Technology, vol. 29, no. 6, pp. 120-123, 2016.

[13] W. Jing, Design of the Broadband Leaky Coaxial Cables, Xi'an University of Electronic Science and Technology, Xi'an, China, 2013.

[14] X. Wang, RF Cable Design Manual, 23rd Research Institute of the Ministry of Electronic Industry, Shanghai Sanshi Communication and Transmission Technology Company, Shanghai, China, 1996.

[15] M. Li, Design of Radiation Leaky Coaxial Cable, Xi'an University of Electronic Science and Technology, Xi'an, China, 2012.

[16] W. Wenyuan, D. Gong, and B. Chen, Antenna Principle, Xi'an University of Electronic Science and Technology Press, Xi'an, China, 2006. 